\title{
Introducing MRF in Patch-based Image Inpainting
}

\author{
Sonali Zatale \\ ME Computer Engineering Department \\ D.Y.P.I.E.T., Pimpri \\ Pune, India
}

\author{
Archana Chaugule \\ Prof. Computer Engineering Department \\ D.Y.P.I.E.T., Pimpri \\ Pune, India
}

\begin{abstract}
The aim of inpainting is to reconstruct the injured or the unwanted portions of the picture, so as to make it appear real. Image inpainting techniques are used to restore the images which get6 damaged due to some reasons. Image inpainting techniques are also used to edit the image so as to remove the unwanted part of the image. Here, an approach of inpainting is proposed which is the patch based image inpainting. Patchbased image inpainting is a technique which uses a top down approach to divide the given image into variable sized blocks. This technique search for the candidate patches of the source region matching to those of the target patches. This approach can be employed to improve the speed and performance of patch-based inpainting method. Objective is to discover ways to remedy the primary faults that afflict digital and scanned photographs, using a combination of algorithms which make the inpainting process faster. To increase performance proposed a novel scheme called MRF (Markov Random Field) is proposed. MRF gives the prior knowledge about the neighboring image patches consistency.
\end{abstract}

\section{Keywords}

Inpainting, patch-based image inpainting, texture features, context-aware, Markov random field modeling.

\section{INTRODUCTION}

Image inpainting is a process of recovering the spoiled images that get damaged due to some scratches or some text or because of some unwanted objects. It is not only used to recover the images but also used to remove unwanted objects from the image. It is very useful technique used in image processing so as to recover the image and to have good quality of spoiled image. It is an art to reconstruct the images.

In image inpainting, the inpainting algorithm fills the missing region by analyzing the whole image and constructs the region by calculating its value from the neighboring pixel value. The application of image inpainting includes image restoration, photo editors, image coding and transmission, virtual restoration of digitalized paintings etc. Consider an example; if the image shows the appearance of text on to the image, because of this text, image is not clearly visible. Hence by using the inpainting techniques this text can be removed and the image can be recovered. Similarly, this concept of the image inpainting can be used to various applications such as Photoshop, image coding, image-editing tools, etc.

As the technology advances, there are various types of data sets that have been used in these technologies, images area also one of the important types of data used widely in the recent technologies. The concept of inpainting allow images to be more adaptable to the required environment. The growing number of images has increased the need for tools which automatically search image collections. While tools based on keywords exist, they have two major drawbacks. Firstly, each image in the collection has to be described by keywords which are extremely time consuming. Secondly, the expressive power of keywords is limited and cannot be exhaustive. Consequently, a significant need for image content based tools exists, for example in stock photo agencies.

\section{FREQUENTLY USED INPAINTING TECHNIQUES}

The image inpainting approaches has been categorised as Diffusion based methods and Patch based methods.

\subsection{Diffusion based techniques}

Diffusion-based method was the first image inpainting technique invented. The approach of this technique was to fill the missing region by diffusing the information of image by analysing the known region pixels. This approach used the pixel level information. This technique is based on the variational method theory and Partial differential method (PDE) theory.

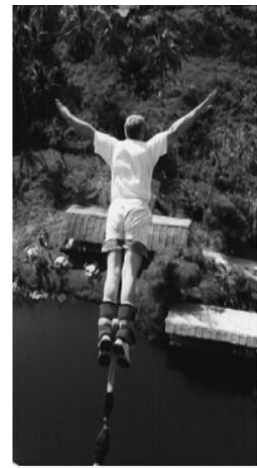

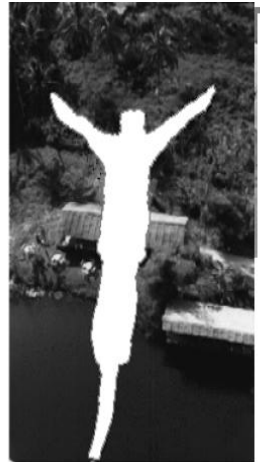

b

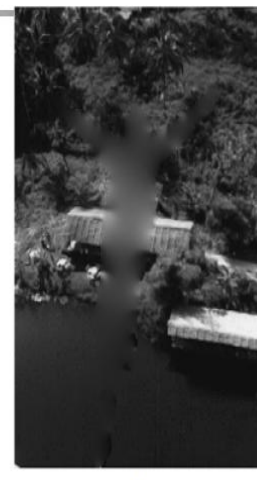

c
Figure 1(a) Original image,(b)selected target region,(c)inpainted image by using diffusion based technique.

In this inpainting technique, the term diffusion belongs to the idea of local information propagation with smoothness constraints. [2]-[5]. This method produces the superb result for the smaller region inpainting. The drawback of this approach is it propagates the linear structures, these linear structure are interrupted by holes and often comes in terms of PDEs. It experiences the difficulties in replication of pixels due their local nature.

\subsection{Patch based techniques}

In Patch-based methods, the image inpainting is done by replacing the missing area by similar patches of that area. In this method, the logic is to search the whole undamaged image for the similar patches relevant to missing area and replace the missing area with the value calculated on the similar patches. In this method, similar patch is called as candidate patches. It is based on the patch based textured synthesis [6], also it uses the structure propagation. As compared to the diffusion based inpainting approaches, the patch based image inpainting approaches are proved to be better with their results, especially in the cases where there require inpainting for larger holes. 


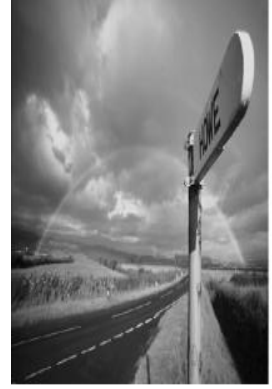

a

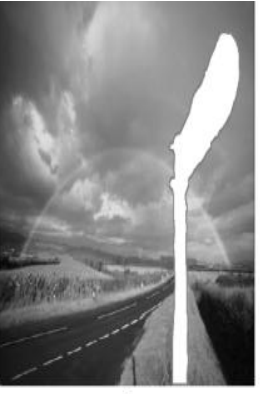

b

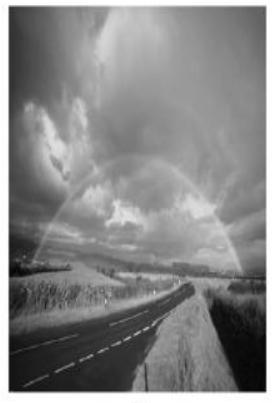

c

Figure 2, (a) Original image, (b) selected target region, (c) inpainted image by using Patch based technique.

Patch-based methods are categorised in to greedy, multiple candidate and global. In greedy, once the target patch is filled with its value obtained from its known pixels, it cannot be changed again [13]. Multiple candidate method deals with the multiple candidate patches combination [10] and weighted average [11], [16] so as to recover the missing region. The third patch based method that is Global method. In this method, inpainting is defined as a global optimization problem. The multiple candidates are termed as labels in this method. A label is chosen such that at all positions, a set of label minimizes a global optimization function. Many of the global methods such as Priority belief propagation (p-BP) [9], structure guided texture inpainting [18], context aware label selection method [20], etc model global image context with Marcov Random Field (MRF) [1]. The main aspect of patch based inpainting approach is to search for the candidate patches. The recent method of patch based inpainting approach is a context aware MRF based inpainting method [1].

\section{RELATED WORK}

Table.1 Related Works in Image Inpainting.

\begin{tabular}{|c|c|c|c|}
\hline Ref. No & Algorithm/methods & Description & Disadvantages \\
\hline 2 & Automatic digital inpainting. & $\begin{array}{l}\text { Algorithm propagates the necessary } \\
\text { information by numerically solving the PDE. } \\
\text { The techniques developed are fully } \\
\text { compatible with the JPEG and JPEG } 2000 \\
\text { standards. }\end{array}$ & $\begin{array}{l}\text { The PDE-based simultaneous filling-in } \\
\text { of textured and structured regions. }\end{array}$ \\
\hline 3 & $\begin{array}{l}\text { Curvature driven } \text { Diffusions } \\
\text { (CDD). }\end{array}$ & $\begin{array}{l}\text { TV inpainting explains some aspects of } \\
\text { human dissoclusion process in vision } \\
\text { psychology. } \\
\text { This proposes inpainting model based on } \\
\text { diffusion mechanism inspired by TV } \\
\text { inpainting aiming at realizing connectivity } \\
\text { principal. }\end{array}$ & $\begin{array}{l}\text { The CDD does not restores even a } \\
\text { single object when the given remaining } \\
\text { disconnected parts are separated by } \\
\text { inpainting domain. }\end{array}$ \\
\hline 4 & $\begin{array}{l}\text { Joint interpolation of the image } \\
\text { gray-levels and gradient / } \\
\text { isophotes directions. }\end{array}$ & $\begin{array}{l}\text { It smoothly extends in an automatic fashion } \\
\text { the isophotes lines into the holes of missing } \\
\text { data. }\end{array}$ & $\begin{array}{l}\text { Need to study ideas for interpolation in } \\
\text { video data }\end{array}$ \\
\hline 7 & Texture synthesis algorithm & $\begin{array}{l}\text { In this approach, patches of the sample } \\
\text { image is copied to the output and then } \\
\text { combined with the optimal image to form a } \\
\text { new output. }\end{array}$ & $\begin{array}{l}\text { It uses approximate offset search that } \\
\text { works in fixed sized patch optimization. }\end{array}$ \\
\hline 8 & $\begin{array}{l}\text { Exemplar based image } \\
\text { inpainting. }\end{array}$ & $\begin{array}{l}\text { A single efficient algorithm achieves } \\
\text { simultaneous propagation of texture and } \\
\text { structure information by combining texture } \\
\text { synthesis and inpainting techniques. }\end{array}$ & $\begin{array}{l}\text { An extension of algorithm to handle } \\
\text { accurate propagation of curved } \\
\text { structures in still photographs as well as } \\
\text { removing objects from video is } \\
\text { required. }\end{array}$ \\
\hline 9 & $\begin{array}{l}\text { Priority belief propagation (p- } \\
\text { BP). }\end{array}$ & $\begin{array}{l}\text { Objective function of this problem is always } \\
\text { well-defined, and corresponds to the energy } \\
\text { of a discrete Markov random field } \\
\text { (MRF).Resolves the BP algorithm } \\
\text { inefficiency in handling MRFs with very } \\
\text { large discrete state spaces. }\end{array}$ & $\begin{array}{l}\text { Unable to test priority-BP algorithm, } \\
\text { this is a generic MRF optimization } \\
\text { scheme, to other labelling problems, as } \\
\text { well, for which the large cardinality of } \\
\text { their state-space causes them to have a } \\
\text { very high computational cost }\end{array}$ \\
\hline 12 & $\begin{array}{l}\text { Robust Image Synthesis by } \\
\text { Adaptive } N \mathrm{D} \text { Tensor Voting }\end{array}$ & $\begin{array}{l}\text { It automatically infers missing information } \\
\text { from a damaged } 2 \mathrm{D} \text { image by tensor voting. } \\
\text { It translates image colour and texture } \\
\text { information into an adaptive ND tensor, } \\
\text { followed by a voting process that infers non- } \\
\text { iteratively the optimal colour values in the } \\
\text { ND texture space for each defective pixel. }\end{array}$ & $\begin{array}{l}\text { If critical information is missing or } \\
\text { insufficient, then it cannot be well } \\
\text { synthesized due to a complex } \\
\text { background and their irregular shapes. }\end{array}$ \\
\hline
\end{tabular}




\begin{tabular}{|l|l|l|l|}
\hline 17 & $\begin{array}{l}\text { Hierarchical Super-Resolution- } \\
\text { Based Inpainting. }\end{array}$ & $\begin{array}{l}\text { A hierarchical super-resolution algorithm is } \\
\text { used to recover details on the missing areas. } \\
\text { The gain is both in terms of computational } \\
\text { complexity and visual quality. }\end{array}$ & $\begin{array}{l}\text { Need to test other SR methods to bring } \\
\text { more robustness to the method. }\end{array}$ \\
\hline 19 & $\begin{array}{l}\text { Markov Random field based } \\
\text { image inpainting with context- } \\
\text { aware label Selection }\end{array}$ & $\begin{array}{l}\text { Context is determined based on the texture } \\
\text { and colour features in fixed image regions } \\
\text { and is used to distinguish areas of similar } \\
\text { content to which the search for candidate } \\
\text { patches is limited. }\end{array}$ & $\begin{array}{l}\text { an alternative to priority belief } \\
\text { propagation. }\end{array}$ \\
\hline
\end{tabular}

\section{PROPOSED WORK}

Although there are various inpainting methods are proposed, T. Ruzic, and A. Pizurica [1] have proposed a new approach of using Marcov Random field concept in the inpainting. The block diagram shown in figure 3 represents the block diagram of proposed work. According to this, the textural descriptors are used to improve the inpainting process. Textural descriptors are defined as the value of a given pixel at given image. It uses two concepts to achieve the better results are: an efficient patch selection strategy and MRF method for efficient inpainting. The first approach works for the selecting the candidate patches for the target patches of inpainting image.

The context aware patch selection approach works as follows:

i. Selects the target area to inpaint.

ii. Selecting the context aware patch.

iii. Dividing the image into blocks of adaptive size by using top down splitting approach.

iv. Assign the contextual descriptors to the image blocks of adaptive sizes.

v. Comparison of contextual descriptors

vi. Selection of most contextually similar block.

The second approach is to apply the MRF modeling to inpainting. It is based on the optimization method so as to be suitable for the MRF inpainting of the images having huge number of labels.

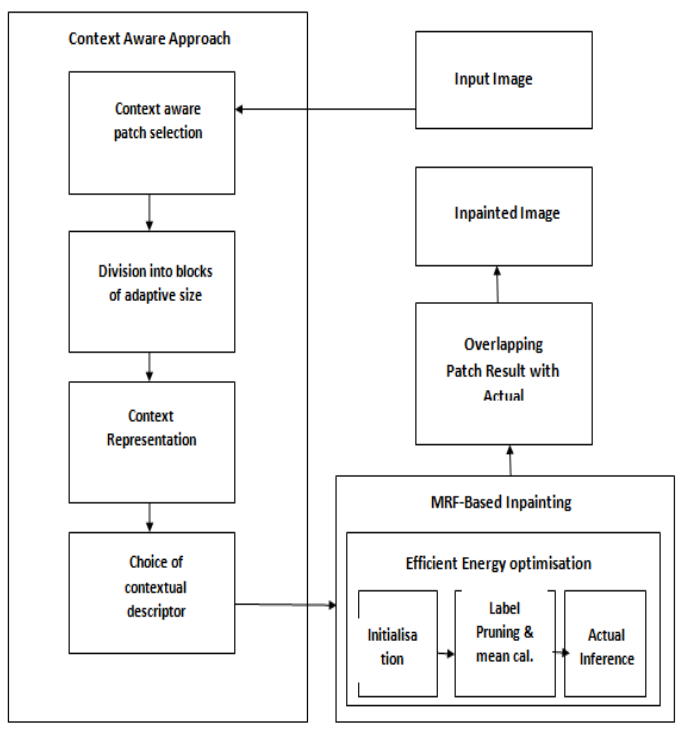

Figure 3: Block diagram of MRF modeling for patch based image inpainting.

\section{RESULT ANALYSIS}

By introducing the concept of MRF in the patch based image inpainting, the differences of the results obtained from both the existing methods and the proposed method can be observed. This section provides the various parameters to distinguish the results such as time and memory. The analysis is given as compared to the existing priority belief propagation method.

\subsection{PSNR}

PSNR stands for peak signal to noise ratio between two images. It is used as one of the quality measure for the images. Higher the values of PSNR better the quality of the image. It is calculated as:

$$
\mathrm{PSNR}=10 \log _{10}\left(\frac{R^{2}}{M S E}\right)
$$

MSE stands for mean squire error.

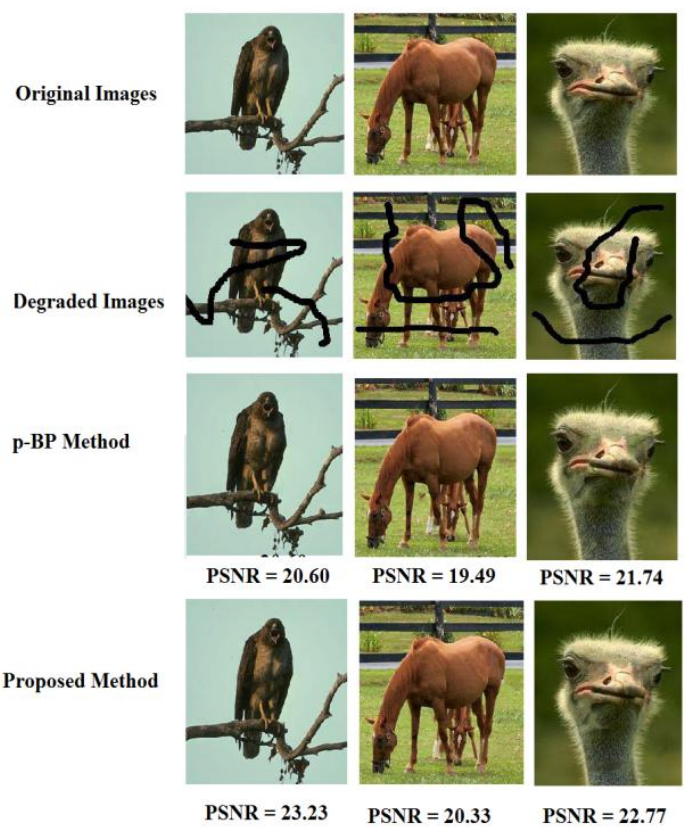

Comparison of PSNR of images for p-BP method and proposed method [1]

\subsection{Time Complexity}

Time complexity can be defined as the amount of time taken to perform the task by the system. It is calculated by:

$$
\text { Total time }=T_{\text {end }}-T_{\text {start }} .
$$

Where $T_{\text {end }}$ represents the time at which the execution ends and $T_{\text {start }}$ represents the time at which the execution start. It gives the total time required by the system to complete the execution 
Figure 5 shows the graph of comparison of existing p-BP method represented by red line and proposed method represented by blue line [20]

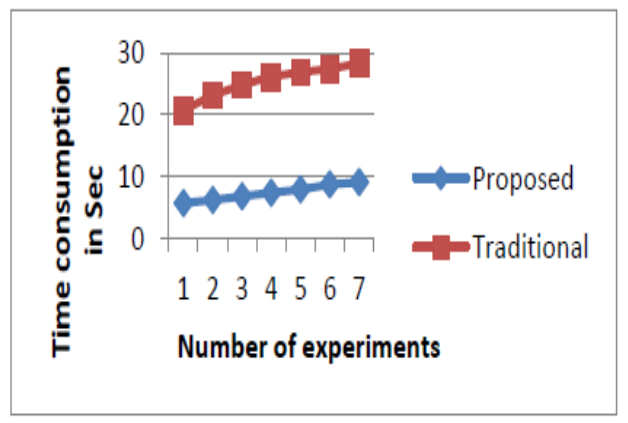

\subsection{Space Complexity}

The minimum amount of memory required to execute the task by the system. The proposed method minimizes both the time complexity and the space complexity.

\section{CONCLUSION}

This paper presents the different techniques for image inpainting, advantages and disadvantages of existing methods / algorithm. Also present the need of the image inpainting techniques. As per the survey, although there exists various inpainting methods still it required to improve the inpainting mechanism so as to achieve desirable outputs. This is achieved by the patch based image inpainting with MRF modeling. This approach proposes a simple and efficient way to perform optimization by first pruning the labels of each node to some small number is also proposed, using both the agreement of the node with its labels and the contextual similarity between regions to which the node and the label belong.

\section{REFERENCES}

[1]. T. Ruzic and A. Pizurica, "Context-aware patch-based image inpainting using Markov random field modeling ," in IEEE Trans.Image Proces., vol. 24, no. 1, pp. 10577149, Jan. 2015.

[2]. M. Bertalmio, G. Sapiro, V. Caselles, and C. Ballester, "Image inpainting,"in Proc. 27th Annu. Conf. SIGGRAPH, New Orleans, LA, USA, 2000, pp. 417424.

[3]. T. F. Chan and J. Shen, "Nontexture inpainting by curvature-driven diffusions," J. Vis. Commun. Image Represent., vol. 12, no. 4, pp. 436-449, 2001.

[4]. C. Ballester, M. Bertalmío, V. Caselles, G. Sapiro, and J. Verdera, "Filling-in by joint interpolation of vector fields and gray levels," IEEE Trans. Image Process., vol. 10, no. 8, pp. 1200-1211, Aug. 2001.

[5]. D. Tschumperlé, "Fast anisotropic smoothing of multivalued images using curvature-preserving PDE's," Int. J. Comput. Vis., vol. 68, no. 1, pp. 65-82, 2006.
[6]. A. A. Efros and W. T. Freeman, "Image quilting for texture synthesis and transfer," in Proc. 28th Annu. Conf. SIGGRAPH, 2001, pp. 341-346.

[7]. V. Kwatra, A. Schödl, I. Essa, G. Turk, and A. Bobick, "Graphcut textures: Image and video synthesis using graph cuts," in Proc. ACM SIGGRAPH, 2003, pp. $277-$ 286.

[8]. A. Criminisi, P. Perez, and K. Toyama, "Region filling and object removal by exemplar-based image inpainting," IEEE Trans. Image Process., vol. 13, no. 9, pp. 1200-1212, Sep. 2004.

[9]. N. Komodakis and G. Tziritas, "Image completion using efficient belief propagation via priority scheduling and dynamic pruning," IEEE Trans. Image Process., vol. 16, no. 11, pp. 2649-2661, Nov. 2007.

[10].Z. $\mathrm{Xu}$ and J. Sun, "Image inpainting by patch propagation using patch sparsity," IEEE Trans. Image Process., vol. 19, no. 5, pp. 1153-1165, May 2010.

[11].O. Le Meur, J. Gautier, and C. Guillemot, "Examplarbased inpainting based on local geometry," in Proc. 18th IEEE ICIP, Sep. 2011, pp. 3462-3465.

[12].J. Jia and C.-K. Tang, "Image repairing: Robust image synthesis by adaptive ND tensor voting," in Proc. IEEE Comput. Soc. Conf. CVPR, Jun. 2003, pp. I-643-I-650.

[13].A. Bugeau, M. Bertalmio, V. Caselles, and G. Sapiro, "A comprehensive framework for image inpainting," IEEE Trans. Image Process., vol. 19, no. 10, pp. 2634-2645, Oct. 2010.

[14]. Anupam P. Goyal, and S. Diwakar, "Fast and enhanced algorithm for exemplar based image inpainting," in Proc. 4th PSIVT, Nov. 2010, pp. 325-330.

[15].T. Ruzic and A. Pizurica, "Texture and color descriptors as a tool for context-aware patch-based image inpainting," Proc. SPIE, Electron. Imag., vol. 8295, pp. 82951P-1-82951P-11, Feb. 2012.

[16].A. Wong and J. Orchard, "A nonlocal-means approach to exemplar-based inpainting," in Proc. 15th IEEE ICIP, Oct. 2008, pp. 2600-2603.

[17].O. Le Meur and C. Guillemot, "Super-resolution-based inpainting," in Proc. 12th ECCV, 2012, pp. 554-567.

[18].H. Ting, S. Chen, J. Liu, and X. Tang, "Image inpainting by global structure and texture propagation," in Proc. 15th ACM Int. Conf. Multimedia, 2007, pp. 517-520.

[19].Y. Pritch, E. Kav Venaki, and S. Peleg, "Shift-map image editing," in Proc. ICCV, 2009, pp. 151-158.

[20].T. Ruzic, A. Pizurica, and W. Philips, "Markov random field based image inpainting with context-aware label selection," in Proc. 19th IEEE ICIP, Sep./Oct. 2012, pp. 1733-1736. 